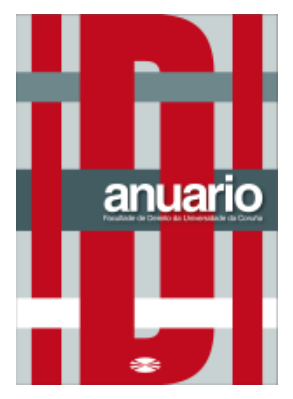

Anuario da Facultade de Dereito da Universidade da Coruña Vol. 22 (2018), pp. 1-20

ISSNe: 2530-6324 || ISSN: 1138-039X

DOI: https://doi.org/10.17979/afdudc.2018.22.0.5001

\title{
LA CONSULTA PREVIA PARA EL USO DE LA TIERRA DESTINADA A LA INDUSTRIA EXTRACTIVA
}

\author{
LUIS JOSÉ BÉJAR RIVERA \\ Profesor Investigador de la Facultad de Derecho de la Universidad Panamericana \\ JUAN MANUEL OTERO VARELA \\ Director Académico de la Maestría en Derecho Administrativo de la Universidad Panamericana \\ CARLOS ALBERTO VILLANUEVA MARTÍNEZ \\ Profesor de Posgrado en la Universidad Panamericana
}

Resumen: Con motivo de una reciente reforma constitucional en materia energética, el sistema jurídico mexicano fue radicalmente modificado, abriendo el mercado energético al sector privado, incluso, incorporando a las compañías públicas al propio mercado, tanto en hidrocarburos como en energía eléctrica. Adicionalmente, tomando en consideración el régimen especial de la tierra en México, mediante el cual se conceden ciertas garantías a los tenedores de ésta, cuando se traten de ejidos o pueblos indígenas el nuevo comportamiento de la industria energética para la explotación de la tierra impone una serie de mecanismos de negociación para el uso del suelo, tomando en consideración el máximo respeto por los derechos y garantías de los pueblos indígenas y de los ejidatarios. Adicionalmente, con una legislación más antigua, se expondrá lo relativo a la consulta previa tratándose de la industria minera en México. Por tanto, el objetivo de este trabajo es exponer de forma general, el régimen de consulta previa que ahora se exige de forma estatutaria para el uso 
de la tierra, sea su destino el sector minero, eléctrico o de hidrocarburos. Se debe tomar en cuenta que la reforma constitucional en materia energética, así como su legislación secundaria, aún y cuando ya entró en vigor, todavía está en proceso de implementación.

Palabras clave: mercado energético, derecho indígena, mecanismos negociación.

Abstract: A propos of the recent constitutional reform enacted in Mexico regarding energy regulation, the legal system was radically changed opening the market to private companies, even fully incorporating the public companies to the market, both in hydrocarbons and electric energy. Also, bearing in mind the special regime of the soil, which grants certain protection to the land owned by indigenous people and/or farmers (Ejidatarios), the new behavior of the energy industry for land exploitation will impose the previous consultation mechanism and other negotiation instruments with the outmost respect for the rights and warranties of indigenous people and ejidatarios. In adition, with and older piece of legislation, we comment on the rights and warraties of those regarding mining industry. Therefore, the objetive of this research is to submit a general depiction of the previous consultation, now enforced on the energy industry for the use of the land. Bear in mind that this constitutional reform and the posterior Acts, are quite recent and still in the process of implementing them by the different legal operators.

Key words: energy regulation, rights of indigenous, negotiation instruments.

SUMARIO: I. INTRODUCCIÓN. II. LOS SUJETOS INVOLUCRADOS. II.1 LOS PUEBLOS INDÍGENAS. II.2 LOS EJIDOS. II.3 LAS EMPRESAS PRODUCTIVAS DEL ESTADO. II.4 LA SECRETARÍA DE ECONOMÍA. III. EL DERECHO A LA CONSULTA PREVIA. III.1 EL ESTADO DE LA CUESTIÓN. III.2 EL CONTENIDO DE LA CONSULTA PREVIA. III.3 REFORMA ENERGÉTICA Y CONSULTA PREVIA. III.3 ¿QUÉ SIGUE DE LA CONSULTA PREVIA? IV. CONSIDERACIONES FINALES. V. FUENTES.

\section{INTRODUCCIÓN}

En el año 2013 se promulgó una reforma constitucional, coloquialmente denominada "Reforma Energética"1, que entre otras cosas abre a la iniciativa privada el sector

\footnotetext{
${ }^{1}$ Formalmente, su denominación es "Decreto por el que se reforman y adicionan diversas disposiciones de la Constitución Política de los Estados Unidos Mexicanos, en Materia de Energía", publicada en el Diario Oficial de la Federación (DOF) el día 20 de diciembre de 2013. Esta reforma forma parte de las llamadas Reformas Estructurales del Estado, entre las cuales se incluyen las reformas en materia de justicia laboral, derecho electoral, reforma política, planeación democrática, competencia económica, telecomunicaciones, en
} 
energético hasta ese momento considerado un área de la economía reservada al Estado, tanto en materia de hidrocarburos como en el sector eléctrico. Esta reforma provocó una serie de modificaciones a la legislación de la materia, así como la expedición de nuevas leyes, que para el objeto de este trabajo estaremos centrando en las disposiciones relativas a la Ley de Hidrocarburos $(\mathrm{LH})^{2}$ y a la Ley de la Industria Eléctrica (LIE) ${ }^{3}$, en las que se introducen, con fuerza estatutaria, los mecanismos de consulta previa, negociación y solución de controversias tratándose del uso de la tierra indispensable para esta industria, originalmente en posesión y/o propiedad de los particulares ${ }^{4}$, pero con un régimen jurídico especial tratándose de los Ejidos $^{5}$ (como titulares de la propiedad social) y los pueblos indígenas o etnias.

Es importante destacar que la figura del ejido corresponde a nuestros orígenes constitucionales, pues es uno de los frutos del movimiento revolucionario de principios del siglo $\mathrm{XX}^{6}$ y que nos diera la Constitución ${ }^{7}$ hoy vigente, la cual estableció como derecho social la tenencia de la tierra, consagrando en su artículo 27, además de la propiedad privada y pública, la propiedad social o ejidal.

Por otra parte, en el año 2001, también mediante reforma constitucional, se estableció en el artículo 2 de la Carta Magna, el reconocimiento expreso del origen multicultural del pueblo mexicano, sustentado en los pueblos indígenas que habitaban el territorio antes de la colonización española, y entre otras cosas, su reconocimiento, garantizando su derecho a la autodeterminación y autonomía, en un marco de unidad nacional.

Justamente, el gran reto de la reforma energética, además mejorar el aprovechamiento de los recursos energéticos mediante la apertura del sector a la iniciativa privada detonando así el desarrollo de infraestructura, es hacerlo acorde con las disposiciones ambientales y en especial, con el total y absoluto respeto a los derechos humanos, particularmente de las comunidades ejidales y los pueblos indígenas y que al día de hoy, no obstante que la

materia de educación, seguridad pública, derecho penal, transparencia y acceso a la información, protección de datos personales, y combate a la corrupción entre otras.

2 Publicada en el DOF el día 11 de agosto de 2014, consultable en http://www.diputados.gob.mx/LeyesBiblio/pdf/LHidro_151116.pdf

3 Publicada en el DOF el día 11 de agosto de 2014, consultable en http://www.diputados.gob.mx/LeyesBiblio/pdf/LIElec_110814.pdf

${ }^{4}$ No es este el espacio para plantear la explicación de las características de esta industria, por lo que nos limitaremos a ofrecer como un dato aceptado, que se trata de no sólo de una industria que opera en red, sino que, dado el estadío tecnológico actual, depende de una infraestructura física que presupone el uso y afectación del suelo (ductos, cables, torres y demás infraestructura para el transporte de los insumos, sean estos petrolíferos o fluido eléctrico).

${ }^{5}$ Un ejido cuenta con personalidad jurídica y patrimonio propios, son propietarios de las tierras que les han dotado o adquirido mediante cualquier título; se rigen por su reglamento interno, sin más limitaciones que las impuestas por la ley. Al respecto, Cfr. Arts. 9 y 10 de la Ley Agraria (LA), publicada en el DOF el día 26 de febrero de 1992, consultable en http://www.diputados.gob.mx/LeyesBiblio/pdf/13_270317.pdf

${ }^{6}$ A mayor abundamiento, Cfr. Ruiz-Massieu, Mario, Derecho Agrario, IIJ-UNAM, México, 1990, pp. 7 y sigs.

7 Publicada en el DOF el día 5 de febrero de 1917, consultable en http://www.diputados.gob.mx/LeyesBiblio/pdf/1_150917.pdf

Anи Fac Der UDC, 2018, 22:1-20 
legislación ya tiene un par de años de haber entrado en vigor, todavía no es del todo operativa.

Es más que evidente el enorme impacto que esta reforma ha provocado y seguirá provocando en el diseño de las políticas públicas de los siguientes años relativas al aprovechamiento adecuado y responsable de los recursos energéticos, su impacto ambiental y trascendencia económica y social, así como respecto de las indemnizaciones en relación al uso de la tierra, tomando en consideración además que, operan tanto normas de derecho público como de derecho privado para garantizar los derecho sociales.

Por lo anterior, el objetivo del presente trabajo es hacer una descripción general de la forma en que está regulada la consulta previa, así como la intervención de los diversos agentes públicos y sus relaciones con las Empresas Productivas del Estado o los concesionarios del uso de la tierra, de cara al aseguramiento del respeto a los derechos fundamentales de los pueblos indígenas y los ejidatarios. Lo mismo vale decir para el caso de la industria minera, pues aun cuando en principio no se vio afectada por la reforma energética (por lo menos de forma directa), los compromisos internacionales obligan al Estado mexicano a recurrir a la figura de la consulta previa también en este caso.

Si bien los estándares internacionales ya establecen la figura de la consulta previa, e incluso existen algunos pronunciamientos aislados del Poder Judicial mexicano al respecto, como ya lo comentamos en líneas precedentes, la reforma energética sigue todavía en proceso de implementación.

Es por ello que en gran medida, la contribución en este trabajo será desde una perspectiva teórica, describiendo el estado de la cuestión en cuanto a la implementación de la legislación especial en materia de consulta previa.

\section{LOS SUJETOS INVOLUCRADOS}

\section{Los Pueblos Indígenas}

Tal y como señalamos en líneas precedentes, México se ha caracterizado por su multiculturalismo, el cual fue reconocimiento en el ámbito constitucional en el año 2001, estableciendo un régimen jurídico especial cuando se trate de comunidades que formen una unidad social, económica y cultural, incluida su lengua ${ }^{8}$, asentadas en un territorio determinado, con autoridades propias de acuerdo a sus usos y costumbres, siempre y cuando exista un respeto absoluto a la unidad nacional y al Estado de Derecho ${ }^{9}$,

\footnotetext{
${ }^{8}$ En términos del artículo 4 de la Ley General de Derechos Lingüísticos de los Pueblos Indígenas (LGDLPI), publicada en el DOF el día 13 de marzo de 2003, consultable en http://www.diputados.gob.mx/LeyesBiblio/pdf/257_171215.pdf, se reconocen, junto con el español todas las lenguas indígenas como lenguas nacionales.

${ }^{9}$ Ilustra lo anterior, la Tesis Aislada XXI.2o.P.A.6 P $\left(10^{\mathrm{a}}\right)$, emitida por el Segundo Tribunal Colegiado en Materia Penal y Administrativa del Vigésimo Primer Circuito, Gaceta del Semanario Judicial de la Federación, Libro 34, Septiembre de 2016, Tomo IV, p. 2655, con la voz: "DERECHO A LA LIBRE DETERMINACIÓN DE LOS PUEBLOS Y COMUNIDADES INDÍGENAS. NO FACULTA A LAS
} 
permitiendo la propiedad y tenencia de la tierra en los términos en que dispone la propia Constitución Política de los Estados Unidos Mexicanos (CPEUM). También cuentan con el derecho de nombrar representantes ante las municipalidades y pleno acceso a la jurisdicción del Estado, tomando en consideración sus usos y costumbres, así como especificidades culturales y a ser asistidos por intérpretes y defensores que tengan conocimiento de su lengua y cultura ${ }^{10}$.

Complementando lo anterior, se puede decir que:

"En términos legales ya no son reconocidos solamente como ciudadanos individuales, sino como colectivos específicos con derechos diferenciados respecto del resto de la ciudadanía. Sus derechos reconocidos a seguir viviendo de una forma distinta de la dominante implican, a la vez, ámbitos de gobierno autónomo y jurisdicción propia.",11

Es obligación del Estado, en sus tres niveles de gobierno (Federación, Estados y Municipios) impulsar el desarrollo regional de las zonas indígenas; mejorar sus condiciones mediante acciones que faciliten el acceso a financiamiento público y privado para ampliar la cobertura de los servicios sociales básicos, así como apoyar sus actividades productivas y para el desarrollo sustentable de su comunidad.

Asimismo, se protegerá la integridad de la tierra de los pueblos indígenas, en términos de lo dispuesto por el párrafo segundo, fracción VII del artículo 27 constitucional.

Para efectos del objeto de este trabajo, es importante destacar lo dispuesto por la fracción IX del Apartado B del artículo 2 de la CPEUM, en la que se señala textualmente:

“Art. 2.- ...

$B \ldots$

IX. Consultar a los pueblos indígenas en la elaboración del Plan Nacional de

Desarrollo y de los planes de las entidades federativas, de los Municipios y, cuando proceda, de las demarcaciones territoriales de la Ciudad de México y, en su caso, incorporar las recomendaciones y propuestas que realicen."

Aun cuando no hace referencia expresa a la figura de la consulta previa, nos parece evidente su vinculación, pues en términos de nuestro sistema jurídico, las acciones de gobierno deberán formar parte del Plan Nacional de Desarrollo (PND) ${ }^{12}$, incluidos por

AUTORIDADES COMUNITARIAS A DICTAR ÓRDENES DE APREHENSIÓN O DE CATEO, POR LO QUE DE HACERLO, LA DETENCIÓN DEL SUPUESTO INCULPADO ES ILEGAL (LEGISLACIÓN DEL ESTADO DE GUERRERO).”

${ }^{10}$ Cfr. Art. 2, Apartado A, fracción VIII de la CPEUM.

11 Sieder, Rachel, Pueblos indígenas $y$ derecho(s) en América Latina, consultado en http://www.rachelsieder.com/wp-content/uploads/2014/01/el-derecho-en-america-latina-rachel_sieder.pdf, el día 23 de febrero de 2018.

${ }^{12}$ Tal como lo exige la Ley de Planeación (LP), publicada en el DOF el día 5 de enero de 1983, consultable en http://www.diputados.gob.mx/LeyesBiblio/pdf/59_281116.pdf, y que en su artículo 3 señala: "Artículo 
supuesto, los proyectos de infraestructura. Adicionalmente, es necesario considerar los compromisos internacionales adquiridos por el Estado mexicano, como es el caso del Convenio 169 de la OIT, sobre derechos de los pueblos indígenas ${ }^{13}$. Aunque se trata de una obligación concurrente para los tres niveles de gobierno, en el ámbito Federal corresponde a la Comisión Nacional para el Desarrollo de los Pueblos Indígenas (CDI) ${ }^{14}$, la implementación práctica de las disposiciones constitucionales a las que hemos hecho mención.

Para dimensionar el peso e importancia de la población indígena en el contexto nacional, el documento Fichas de Información Básica de la Población Indígena, 2015 ${ }^{15}$, emitido por la propia CNDI, señala que en México había en dicho año, 12`025,947 personas consideradas como indígenas ${ }^{16}$, lo que representaba el $10.1 \%$ de la población; sin embargo, si se considera a la población autoadscrita ${ }^{17}$ su número se incrementa a $25^{\prime} 694,928$, que representa el $21.5 \%$ de la población nacional. Sólo a manera de ejemplo, las mayores concentraciones de población indígena autoadscritas se ubican en los estados de Oaxaca, con 65.7\%; Yucatán, con 65.4\%; Campeche, con 44.5\%; Quinta Roo con 44.4\%; Hidalgo, con 36.2\%; Chiapas, con 36.1\%; Puebla con 35.3\%, y Guerrero, con 33.9\%.

\section{Los Ejidos}

3o.- Para los efectos de esta Ley se entiende por planeación nacional de desarrollo la ordenación racional y sistemática de acciones que, en base al ejercicio de las atribuciones del Ejecutivo Federal en materia de regulación y promoción de la actividad económica, social, politica, cultural, de protección al ambiente y aprovechamiento racional de los recursos naturales así como de ordenamiento territorial de los asentamientos humanos y desarrollo urbano, tiene como propósito la transformación de la realidad del país, de conformidad con las normas, principios y objetivos que la propia Constitución y la ley establecen. Mediante la planeación se fijarán objetivos, metas, estrategias y prioridades, así como criterios basados en estudios de factibilidad cultural; se asignarán recursos, responsabilidades y tiempos de ejecución, se coordinarán acciones y se evaluarán resultados."

13 Consultable en http://www.ilo.org/wcmsp5/groups/public/---americas/---rolima/documents/publication/wcms_345065.pdf

${ }^{14}$ La CDI se define legalmente como un organismo descentralizado, no sectorizado, y tiene por objeto orientar, coordinar, promover, apoyar, fomentar, dar seguimiento y evaluar los programas, proyectos, estrategias y acciones públicas para el desarrollo integral y sustentable de los pueblos y comunidades indígenas, para lolo cual se le otorgan diversas facultades en términos de la Ley de la Comisión Nacional para el Desarrollo de los Pueblos Indígenas (LCDI), publicada en el DOF el día 21 de mayo de 2003, consultable en http://www.diputados.gob.mx/LeyesBiblio/pdf/261_220617.pdf

15 El documento puede ser consultado en la siguiente dirección electrónica: http://www.cdi.gob.mx/indicadores2015/

${ }^{16}$ Según se señala en la introducción del documento en cita, la metodología empleada tiene como base la identificación del hogar indígena, que se define como aquel donde el jefe(a) del hogar, cónyuge o alguno de los ascendientes (madre o padre, madrastra o padrastro, abuelo(a), bisabuelo(a), tatarabuelo(a), suegro(a)), declararon ser hablantes de alguna lengua indígena, caso en el cual, todos los integrantes de esos hogares fueron contabilizados como población indígena. A dicho volumen se adicionaron aquellas personas que declararon hablar alguna lengua indígena y que no forma parte de estos hogares.

${ }^{17}$ Siempre de acuerdo con la introducción del documento de mérito, se señala que por primera vez se incluyó en el cuestionario básico de la Encuesta Intercensal 2015, una pregunta para identificar a las personas que se autoadscriben como indígenas, lo que permitió identificar el segmento de la población nacional que se considera indígena aun cuando en el registro de datos ya no se encuentre asociado a ser hablante de alguna legua indígena. 
En términos de lo dispuesto por el artículo 27 de la CPEUM, la Nación ejerce la propiedad originaria sobre todo el territorio nacional, y el dominio directo sobre la totalidad de los recursos naturales.

De la propiedad originaria se deriva la propiedad privada en el Estado mexicano, así como la llamada propiedad social, es decir, los Ejidos como un régimen jurídico de protección especial para los tenedores de las tierras destinadas al aprovechamiento de los recursos y asentamientos humanos de las comunidades ejidales ${ }^{18}$, y cuya propiedad solo puede ser afectada ya sea por decisión de la Asamblea ejidal (tratándose de conversión de la tierra en propiedad privada) o bien, mediante expropiación por causa de utilidad pública ${ }^{19}$.

En cifras, al año 2016, se contaba con 29,690 ejidos registrados a nivel nacional ${ }^{20}$ que representan una superficie en hectáreas de $82,559,746.938873^{21}$, con un total de 2,392 comunidades registradas $^{22}$

Reiterando lo señalado en líneas precedentes, la figura de la propiedad social así como el régimen jurídico especial de los ejidos representa uno de los triunfos del movimiento revolucionario de principios del siglo $\mathrm{XX}$, dando un carisma de corte socialista a nuestra Constitución (junto con el derecho laboral, la seguridad social y el derecho a la educación), y como objeto de estudio para el derecho, ha evolucionado hacia un derecho especial, el derecho agrario, dedicado a éste régimen legal, estableciendo además autoridades específicas para la atención de este tema, como lo son la Secretaría del Derecho Urbano y Territorial (SEDATU), antes Secretaria de la Reforma Agraria, la Procuraduría Agraria, el Registro Agrario Nacional, y un Tribunal administrativo para la solución de conflictos en la materia, el Tribunal Agrario.

Al igual que con los pueblos indígenas, se puede decir que el ejido se entiende como una unidad jurídica y colectiva de personas, que han sido dotadas de tierra para su explotación y aprovechamiento con vocación agropecuaria, fruto del reparto territorial derivado del movimiento revolucionario de principios del siglo XX.

\section{Las Empresas Productivas del Estado}

En el apartado anterior señalamos que el dominio directo sobre los recursos naturales le corresponde al Estado, y sólo podrán ser explotados por particulares mediante concesión, excepto tratándose del petróleo y demás hidrocarburos, así como de los servicios públicos

\footnotetext{
${ }^{18}$ Cfr. Fracción VII del art. 27 de la CPEUM.

${ }^{19}$ En términos de lo dispuesto por el artículo 93 de la LA, así como por lo señalado en el diverso 1 de la Ley de Expropiación, publicada en el DOF el día 25 de noviembre de 1936, consultable en http://www.diputados.gob.mx/LeyesBiblio/pdf/35.pdf

${ }^{20}$ Consultable en http://www.ran.gob.mx/ran/indic_bps/1_ER-2016.pdf

${ }^{21}$ Consultable en http://www.ran.gob.mx/ran/indic_bps/2_SER-2016.pdf

${ }^{22}$ http://www.ran.gob.mx/ran/indic_bps/16_CR-2016.pdf
} 
de trasmisión y de distribución de energía eléctrica. En materia minera, no se autorizan concesiones tratándose de minerales radioactivos ${ }^{23}$.

Históricamente en nuestro país, desde la llamada Expropiación Petrolera en los años 30 del siglo pasado, el sector hidrocarburos le corresponde exclusivamente a la Nación por medio de su organismo descentralizado Petróleos Mexicanos (PEMEX).

Lo mismo se puede decir de la actividad relacionada con el sector eléctrico, nacionalizado en 1960, siendo desde entonces hasta la reforma constitucional de 2013, una actividad desarrollada por diversos organismos descentralizados hasta quedar integrados en su totalidad en la Comisión Federal de Electricidad (CFE).

Sin embargo, con motivo de la reforma energética del año 2013, se establece que la participación estatal en los sectores de hidrocarburos y eléctrico, se realizará por conducto de las Empresas Productivas del Estado (EPE) ${ }^{24}$; y aun cuando se prohíbe expresamente el otorgamiento de concesiones en estas materias, si se permite la participación privada a partir de contratos entre el Estado y los particulares o las propias EPEs ${ }^{25}$.

De este forma, en un proceso de reingeniería constitucional y legislativa, PEMEX y CFE de ser considerados organismos públicos descentralizados, reguladas por la Ley Federal de Entidades Paraestatales (LFEP) ${ }^{26}$ se convierten en EPEs, teniendo como características: a) Cuentan con personalidad jurídica propia, b) Cuentan con patrimonio propio, c) Cuentan con autonomía técnica, operativa y de gestión, d) Se rigen por su ley especial ${ }^{27}$, y la supletoriedad le corresponde al derecho mercantil y en su defecto al civil y, e) Las normas siempre serán interpretadas a su favor en relación al cumplimiento de su objeto.

Aún cuando guardan enorme parecido con los organismos descentralizados ${ }^{28}$ podemos destacar que la gran diferencia que existe entre estos y las EPEs radica en qué estas últimas tienen como objeto principal el de generar recursos para el Estado, permitiendo así que mayores ingresos para el erario público, así como garantizar una mejor explotación de los hidrocarburos y de la industria eléctrica ${ }^{29}$.

\footnotetext{
${ }^{23}$ Cfr. Párrafos quinto y sexto del art. 27 de la CPEUM.

${ }^{24}$ Cfr. Párrafo tercero del art. 25 de la CPEUM.

${ }^{25}$ Cfr. Párrafo quinto del art. 27 de la CPEUM en materia de energía eléctrica y Párrafo sexto del mismo numeral en materia de hidrocarburos, pero en todo caso, expresamente señalando que la planeación y el control del sistema eléctrico nacional, así como los servicios públicos de transmisión y de distribución de energía eléctrica y la propiedad del petróleo y los hidrocarburos es exclusiva de la Nación.

26 Publicada en el DOF el día 14 de mayo de 1986, consultable en http://www.diputados.gob.mx/LeyesBiblio/pdf/110_181215.pdf

${ }^{27}$ Para PEMEX, la Ley de Petróleos Mexicanos (LPEMEX), publicada en el DOF el día 11 de agosto de 2014, consultable en http://www.diputados.gob.mx/LeyesBiblio/pdf/LPM_110814.pdf; para CFE, la Ley de la Comisión Federal de Electricidad (LCFE), publicada en el DOF el mismo día y consultable en http://www.diputados.gob.mx/LeyesBiblio/pdf/LCFE_110814.pdf

${ }^{28}$ A mayor abundamiento en cuanto a sus características, Cfr. Béjar Rivera, Luis José, Curso de Derecho Administrativo, Novum, México, 2012, pp. 174-186.

${ }^{29}$ Para una descripción más detallada de las características de estas empresas, Cfr. Villanueva Martínez, Carlos, "La nueva regulación del sector eléctrico en México. Una visión panorámica de su marco jurídico", en
} 
Es importante señalar en este punto que las EPEs no son los únicos actores gubernativos que intervienen tratándose de las consultas previas en relación a la industria energética, sin embargo, tratar de señalarlos a todos en este punto desviaría el objeto de estudio del presente trabajo.

\section{La Secretaría de Economía}

La Secretaría de Economía, órgano centralizado de la Administración Pública Federal es la responsable legalmente de aplicar la Ley Minera $(\mathrm{LM})^{30}$, y entre sus atribuciones destacamos la facultad de otorgar las concesiones y asignaciones mineras a los particulares en relación a los minerales susceptibles de explotación por los particulares ${ }^{31}$.

En términos generales, la Secretaría será la responsable de las concesiones y asignaciones, los cuales pueden ser otorgadas por acto discrecional, o bien, mediante concurso.

Es importante señalar que en términos de la LM, cuando una comunidad indígena esté asentada en un terreno con vocación minera, siempre tendrá preferencia para ser concesionaria de la mina ${ }^{32}$. Asimismo, tratándose del concurso para el otorgamiento de concesiones, si el terreno se encuentra en un área habitada y ocupada por una comunidad indígena, ésta tendrá el derecho de igualar la mejor oferta y obtendrá el derecho preferente sobre la concesión ${ }^{33}$.

\section{EL DERECHO A LA CONSULTA PREVIA}

\section{El Estado de la cuestión}

El derecho mexicano siempre se ha contado con una legislación especial, particularmente proteccionista tratándose de los ejidos y los derechos ejidales ${ }^{34}$ y que en el caso de la legislación indigenista, introduce una serie de garantías a favor de los pueblos autóctonos a partir de la reforma constitucional de 2001. Desafortunadamente, no podemos afirmar que esto haya sido suficiente al día de hoy.

\footnotetext{
Moreno Castillo, Luis Ferney y Hernández-Mendible, Víctor Rafael [Coordinadores], Derecho de la Energía en América Latina. Tomo II, Universidad Externado de Colombia, Bogotá, Colombia 2017, pp. 220-231.

30 Publicada en el DOF el día 26 de junio de 1992, consultable en http://www.diputados.gob.mx/LeyesBiblio/pdf/151_110814.pdf

${ }^{31}$ Cfr. Art. 4 de la LM.

${ }^{32}$ Cfr. Art. 13, párrafo tercero de la LM.

${ }^{33}$ Cfr. Art. 13 BIS, párrafo de la LM.

${ }^{34}$ Como muestra de lo anterior, la legislación de Amparo, tratándose del Amparo en materia agraria históricamente ha otorgado una serie de protecciones legales, incluso la suplencia de la queja deficiente cuando se importe peligro sobre derechos ejidales. Al respecto, Cfr. Artículos 17, fracción III, artículo 79, fracción IV, 75 o el diverso 79, fracción IV de la Ley de Amparo, publicada en el DOF el día 2 de abril de 2013, consultable en http://www.diputados.gob.mx/LeyesBiblio/pdf/LAmp_190118.pdf, solo por citar algunos ejemplos.
} 
A este respecto, cabe mencionar que recientemente se dio a conocer el documento Compendio de información que presentan la Coalición de Organizaciones de la Sociedad Civil al Grupo de Trabajo sobre Empresas y Derechos Humanos de la ONU, en el que se documentan casos específicos de supuestas violaciones a los Derechos Humanos en materia de proyectos de energía hidroeléctrica y eólica, que se imputan tanto a empresas públicas como privadas $^{35}$.

Sin embargo, como señalamos en líneas precedentes, aun cuando México ha adquirido una serie de compromisos internacionales respecto de la consulta previa, es poco lo que se había legislado al respecto, y no es sino a partir de la reforma energética que formalmente se introduce a nuestro sistema.

\section{Así, César Rodríguez Garavito señala que:}

"La consulta previa a pueblos indígenas y grupos étnicos se ha convertido en uno de los temas más difíciles y controvertidos del derecho nacional e internacional de los derechos humanos. En efecto, en apenas dos décadas, desde la adopción del Convenio 169 de la Organización Internacional del Trabajo en 1989, pasó de ser un asunto relativamente especializado e invisible a ser el objeto de conflictos jurídicos, políticos y sociales en los que se juegan tanto intereses económicos como la supervivencia de pueblos indigenas y otras comunidades étnicas alrededor del mundo. ",36

En este sentido, el Poder Judicial Federal ha sido sensible y bajo su auspicio se publicó el documento titulado Protocolo de Actuación para quienes imparten justicia en casos relacionados con Proyectos de Desarrollo e Infraestructura, emitido por la Suprema Corte de Justicia de la Nación (SCJN) en el que se afirma que:

\footnotetext{
${ }^{35}$ El documento de mérito incluye fichas de diversos proyectos, en los que asegura, se han violado derechos humanos y se ha criminalizado a los defensores de éstos. Los proyectos son la construcción de la represa hidroeléctrica Chicoasen II; la Presa La Parota y la criminalización del Consejo de Ejidos y Comunidades Opositoras a dicho proyecto; las Comunidades del Pueblo Tutunaku contra el Proyecto Hidroeléctrico Puebla 1; el caso de la Presa Hidroeléctrica Las Cruces; el Parque Eólico de Eólica del Golfo 1; el Comité de Resistencia al Proyecto Eólico de Unión Hidalgo, así como el Energía Eólica del Sur, casos en los cuales se habla de la afectación de comunidades indígenas, de la violación al derecho a la consulta previa y la criminalización de los defensores tanto por parte de autoridades como de particulares permisionarios, y en los que la pretensión consiste en la suspensión de la ejecución de los proyectos. Sin embargo, se incluye una ficha relativa al caso de la Red Nacional de Resistencia Civil, organización que protesta por las tarifas eléctricas y el corte del servicio, alegando un derecho humano a la energía. Ello pone de manifiesto la versatilidad y extensión de los derechos humanos, que lo mismo se invocan para oponerse a los proyectos que permitirían un abasto más amplio y económico de energía, que para reclamar el acceso a la energía como un derecho de esta índole. Al respecto, véase el documento de mérito, pp. 81-95.

${ }^{36}$ Rodríguez Garavito, César (Ed.), "La consulta previa a pueblos indígenas: los estándares del derecho internacional", en Programa de Justicia Global y Derechos Humanos, Documentos No. 2, 2009, p. 5, consultable http://www.libertadciudadana.org/archivos/Biblioteca\%20Virtual/Documentos\%20Informes\%20Indigenas/D ocumentos\%20Internacionales/Derecho/Consulta\%20Previa\%20a\%20Pueblos\%20Indigenas\%20Los\%20Esta ndares\%20del\%20Derecho\%20Internacional\%202009.pdf
} 
"[...] el marco normativo vigente y la conflictividad social señalada por las y los relatores internacionales [...] derivada de la afectación a derechos de poblaciones específicas por la implementación de proyectos a gran escala, permite prever que los Poderes Judiciales del país tendrán cada vez más casos relacionados con posibles violaciones a derechos humanos resultado de proyectos de desarrollo e infraestructura. ${ }^{, 37}$

Ahora bien, es de destacar la desconfianza o reserva que el documento expresa respecto del interés general como justificación de estos proyectos, cuando afirma: "Aun cuando estos proyectos efectivamente conlleven lo que presumen, ideas como el bienestar general, más aún cuando son proyecciones y no están fundadas en hechos verificables, no pueden llevar a la realización de dichos proyectos sin tomar en cuenta las posibles afectaciones que pueden significar" ${ }^{38}$ y expresa claramente su inclinación por la preponderancia de los derechos humanos:

"El hecho de que tanto los posibles beneficios de un proyecto de desarrollo o infraestructura como las afectaciones que éstos pueden desencadenar se traduzcan en derechos de personas o colectivos, significa que el debate sobre la viabilidad o no de un proyecto de desarrollo debe darse a partir del análisis de la situación que suponga mayores beneficios para los derechos humanos y menos limitaciones a estos derechos. "39

Destaca también el expreso reconocimiento del derecho a la consulta, al señalarse que "[...] no podría impulsarse ningún tipo de proyecto de desarrollo o infraestructura sin haberlo consultado con las personas afectadas. "40

Finalmente, debe señalarse que entre los derechos humanos susceptibles de ser afectados se mencionan, de forma específica, los derechos a la participación y a la consulta, respecto de los cuales se sugieren, entre otras consideraciones que deben ser tomadas en cuenta por los juzgadores, verificar que cuando los afectados exijan participar en la toma de decisiones relativas a un proyecto, las autoridades abran los cauces institucionales apropiados para ello; que cuando no sea convocada una consulta a pesar de haber sido solicitada o exista un vicio de procedimiento en su celebración, puedan detener el proceso y asegurarse de que la consulta sea realizada; revisar si las leyes, normas o algún otro instrumento general violenta el derecho a una consulta libre, previa e informada de los pueblos y comunidades indígenas; revisar si la aprobación de un proyecto específico respetó este derecho a la consulta de pueblos y comunidades indígenas, así como revisar si una ley, norma o algún otro instrumento general contiene las garantías procesales para respetar y proteger este derecho y en caso contrario, analizar el otorgar modificaciones procedimentales para asegurar su respeto ${ }^{41}$.

\footnotetext{
${ }^{37}$ Gutiérrez Rivas, Rodrigo y María Silvia Emanuelli (Coordinadores), Protocolo de Actuación para quienes imparten justicia en casos relacionados con Proyectos de Desarrollo e Infraestructura, Suprema Corte de Justicia de la Nación, México 2014, p. 17.

${ }^{38}$ Ibidem, p. 12.

${ }^{39}$ Ibíd.

${ }^{40}$ Ibid.

${ }^{41}$ Ibidem pp. 85-86.
} 
En este sentido, encontramos que ya se han producido algunos pronunciamientos judiciales, como es el caso de la Segunda Sala de nuestra SCJN con el siguiente criterio:

\section{"PUEBLOS Y COMUNIDADES INDÍGENAS. DERECHO A SER CONSULTADOS. REQUISITOS ESENCIALES PARA SU} CUMPLIMIENTO.- De conformidad con los estándares internacionales en materia de protección a los derechos de las comunidades indígenas, las características específicas del procedimiento de consulta variarán necesariamente en función de la naturaleza de la medida propuesta y del impacto sobre los grupos indígenas, por lo que los jueces deberán analizar en cada caso concreto si el proceso de consulta realizado por las autoridades cumple con los estándares de ser: a) previa al acto, toda vez que debe llevarse a cabo durante la fase de planificación del proyecto, con suficiente antelación al comienzo de las actividades de ejecución; b) culturalmente adecuada, ya que debe respetar sus costumbres y tradiciones, considerando en todo momento los métodos tradicionales que utilizan en la toma de sus decisiones; en ese sentido, las decisiones que las comunidades indígenas tomen de acuerdo con el ejercicio de sus usos y costumbres deben respetarse en todo momento, lo que implica que las autoridades deben llevar a cabo la consulta, a través de medios e instrumentos idóneos para las comunidades indígenas, de suerte que la falta de acceso a las tecnologías de la información, no signifique un menoscabo en el ejercicio de este derecho; c) informada, al exigir la existencia de información precisa sobre la naturaleza y consecuencias del proyecto, debiendo adoptar todas las medidas necesarias para que sea comprensible, por lo que si así lo requiere el caso concreto, deberá ser proporcionada en las lenguas o idiomas de las comunidades o pueblos involucrados, así como con todos los elementos necesarios para su entendimiento, de manera que los tecnicismos científicos no constituyan una barrera para que las comunidades puedan emitir una opinión; $y$ d) de buena fe, pues la consulta exige la ausencia de cualquier tipo de coerción por parte del Estado o de particulares que actúen con su autorización o aquiescencia. Asimismo, debe efectuarse fuera de un ambiente hostil que obligue a las comunidades o pueblos indígenas a tomar una decisión viciada o precipitada. ",42

En esta línea de pensamiento, la Comisión Nacional de los Derechos Humanos (CNDH) tratándose de desarrollo de proyectos de infraestructura y derechos de los pueblos indígenas ha manifestado: "Tanto el artículo 2o. constitucional como la Declaración de Naciones Unidas sobre los Derechos de los Pueblos Indígenas y el Convenio 169 de la OIT reconocen el derecho de estos pueblos a ser consultados en aquellos asuntos que les afectan, al igual que a ser partícipes de los beneficios que estos desarrollos generen ",43, y

\footnotetext{
42 Tesis Aislada 2a.XXIX/2016 (10 a), visible en la GSJF, Libro 31, Junio de 2016, Tomo II, p. 1212.

${ }^{43}$ Melesio Nolasco, Josefina María de la Soledad, Megaproyectos y derechos humanos de los pueblos indígenas, Comisión Nacional de los Derechos Humanos, México 2014, pp. 9-10.
} 
como justificación del reconocimiento de este derecho a la consulta, plantea que " [...] los proyectos de desarrollo económico, ajenos a las culturas indígenas y basados en la explotación de sus recursos naturales han generado fuertes demandas de los pueblos indígenas al Estado; exigen que se garantice su derecho a ser consultados ante la ejecución de proyectos tales como la adjudicación de tierras para la construcción de plantas hidroeléctricas, la explotación minera, reacomodos de poblaciones indígenas, explotación de recursos acuíferos y la explotación del turismo, entre otros. "44

Consecuentemente, el documento afirma que con la imposición de megaproyectos en nombre del desarrollo del país, se trastoca la trama cultural, dado que el territorio es clave en la reproducción cultural de un pueblo, y que este tipo de proyectos no sólo desplaza a los pueblos indígenas, sino que los despoja de su territorio y de sus recursos naturales, sin que sean beneficiados por dichos proyectos ${ }^{45}$.

\section{El contenido de la consulta previa}

Siguiendo la Recomendación General No. 27/2016"6: "Sobre el derecho a la consulta previa de los pueblos y comunidades indígenas de la República Mexicana” de la CNDH, el contenido de las consultas deben tener como características: a) ser conforme a un principio de buena fe (fundadas en la honestidad y el respeto a las diferencias); b) con participación efectiva (las comunidades definen quiénes participan en las consultas); c) a través de instituciones representativas (considerando sus formas de organización tradicionales); d) con un mecanismo culturalmente adecuado (métodos y procedimientos que faciliten y permitan la participación y la comunicación, considerando lengua, información previa, completa y oportuna, etc.), y e) a través de instituciones reconocidas (por los pueblos y comunidades indígenas y las instancias gubernamentales y no gubernamentales que participen $)^{47}$.

En dicha recomendación, dirigida al Titular del Poder Ejecutivo Federal, Congreso de la Unión, Gobernadores, Jefe de Gobierno de la Ciudad de México y Poderes Legislativos de las Entidades Federativas de la República Mexicana. Esencialmente, el objeto de la recomendación consiste en solicitar la expedición de una ley sobre el derecho de consulta previa, que resulte transversal a los rubros que se vean afectados, asegurando la participación de los pueblos indígenas, así como de organizaciones civiles y académicas en el diseño de estas normas.

Si bien es cierto que la Recomendación no define el derecho a la consulta previa, libre e informada, lo califica como un: "[...] principio general del derecho internacional y como un derecho humano colectivo de los pueblos y comunidades indígenas [...] ",4 , por lo cual

\footnotetext{
${ }^{44}$ Ibidem, p. 9.

45 Ibidem, p. 11.

${ }^{46}$ Publicada en el DOF el día 12 de agosto de 2016, consultable en http://www.cndh.org.mx/sites/all/doc/Recomendaciones/generales/RecGral_027.pdf

${ }^{47}$ Cfr. Melesio Nolasco, Josefina María de la Soledad, Op. Cit., p. 13.

${ }^{48}$ Numeral 44 de la Recomendación 27/2016 CNDH.
} 
estima que: "[...] cobra vital relevancia que los grupos interesados, tengan acceso a un recurso mediante el cual se garantice el respeto de sus derechos frente a las acciones estatales que pueden llegar a conculcárselos [...]" ${ }^{\text {49 }}$; pero no acota esta obligación exclusivamente al ámbito de la acción estatal, sino que determina que: "[...] para garantizar la protección de los derechos de la comunidad en el contexto de actividades empresariales, el Estado debe adoptar medidas para observar, fiscalizar y monitorear la participación de la empresa."

De acuerdo con la Recomendación, el derecho a la consulta tiene al menos 5 características:

1) Su carácter previo obliga al Estado a: "[...] efectuar un acercamiento desde etapas tempranas del proyecto, como forma de asegurar la participación e incidencia de la comunidad en los actos del Estado que pudieran llegar a afectarles [...]"51 En proyectos de largo plazo debe garantizarse la participación de las comunidades en todas las fases de diseño, ejecución y evaluación. ${ }^{52}$

2) Su carácter libre, que significa que se desarrolle libre de interferencias externas y exento de coerción, intimidación y manipulación. ${ }^{53}$

3) $\mathrm{Su}$ carácter de informada consiste en "[...] proveer a las comunidades que serán afectadas, de información completa, comprensible, veraz y suficiente, que les permita adoptar una decisión adecuada a sus necesidades" $" 54$. La consulta deberá adoptar un modelo de verdadera asociación, a través de acuerdos que garanticen la protección de los derechos del pueblo indígena interesado, que deberá participar en la toma de decisiones y de los beneficios ${ }^{55}$.

4) Para que se considere de buena fe, "[...] deben evitarse toda clase de acciones tendientes a intimidar, hostigar, amenazar o crear un clima de tensión y desintegración social entre los sujetos de la consulta, mediante la corrupción de los líderes comunales o del establecimiento de liderazgos paralelos "

5) Procedimientos culturalmente adecuados entendidos como "[...] los que usan los pueblos para discutir sus asuntos" 57. La participación indígena implica dos obligaciones: primero, hacerlos participes y atender sus opiniones de acuerdo a sus tradiciones y costumbres, y segundo, sentar las bases para que las comunidades puedan ser parte de manera efectiva, informada y libremente en el respectivo procedimiento administrativo, legislativo o de otra índole que puedan incidir en sus intereses o derechos ${ }^{58}$.

\footnotetext{
49 Ibidem, Numeral 46

${ }^{50}$ Ibidem, Numeral 48

${ }^{51}$ Cfr. Ibidem, Numeral 55

${ }^{52}$ Cfr. Ibidem, Numeral 58

${ }^{53}$ Cfr. Ibidem, Numeral 60

${ }^{54}$ Cfr. Ibidem, Numeral 62

${ }^{55}$ Cfr. Ibídem, Numeral 66.

${ }^{56}$ Cfr. Ibídem, Numeral 68.

${ }^{57}$ Cfr. Ibídem, Numeral 72

${ }^{58}$ Cfr. Ibídem, Numeral 79.
} 
Agotar el contenido de esta Recomendación emitida por la CNDH resultaría un trabajo interminable, que excede los límites del presente documento, pero vale la pena destacar la posición que se asume en relación al resultado de la consulta, es decir, si se considera vinculatorio (para las partes y agentes involucrados) su resultado, independientemente de su sentido.

En este tenor, se debe aclarar que la Recomendación de mérito explícitamente plantea la necesidad de obtener el consentimiento, por lo que no se debe confundir consulta con consentimiento, ya que claramente el resultado de una consulta previa no necesariamente lleva al consentimiento de los pueblos y comunidades involucradas, y esto implica que para el desarrollo de los proyectos de infraestructura, se debe contar con el consentimiento de los pueblos ${ }^{59}$; sin embargo, en caso de no obtenerse y que continúe la necesidad del desarrollo del proyecto, se deberá demostrar que la medida es necesaria, proporcional, tiene un objetivo legítimo en un sociedad democrática y que en todo caso, se tomarán las medidas necesarias para disminuir el impacto sobre los derechos de los pueblos indígenas y hacer las reparaciones del daño necesarias ${ }^{60}$.

\section{Reforma energética y consulta previa}

Ya señalamos en líneas precedentes que en virtud de la reforma energética del año 2013 se transformó el sector con el fin de permitir una mayor apertura de mercado y competitividad así como un mejor y más eficiente aprovechamiento de los recursos energéticos, modificando estructuras administrativas existentes y creando otras tantas ${ }^{61}$. También debemos señalar que esta reforma, ha sido considerada por algún sector de la sociedad civil como violatoria de derechos humanos, ya que contradice el derecho de consulta y la pluriculturalidad al ignorar los impactos sociales y ambientales negativos para las comunidades indígenas por la explotación de recursos naturales ${ }^{62}$. En contraposición, la postura oficial plantea que es una reforma que tomó en consideración todos esos elementos, afirmando que se transforma de fondo a un sector que estaba reservado exclusivamente al monopolio público, para abrirse a la competencia y al sector privado ${ }^{63}$.

Entrando en materia, la LIE y la LH expresamente señalan que para el desarrollo de proyectos de infraestructura en la industria eléctrica o de hidrocarburos (públicos o privados), se debe atender a los principios de sostenibilidad y respeto a los derechos humanos de las comunidades y pueblos de las regiones donde se pretendan desarrollar ${ }^{64}$.

\footnotetext{
${ }^{59}$ Cfr. Ibídem, Numeral 156.

${ }^{60}$ Cfr. Ibidem, Numeral 159.

${ }^{61}$ A mayor abundamiento, Cfr. Béjar Rivera, Luis José, "La nueva organización administrativa en el sector energético" en Derecho de la Energía en América Latina (Moreno Castillo, Luis Ferney \& Hernández Mendible, Víctor Rafael (Coords.), t. II, Universidad Externado de Colombia, Bogotá, 2017.

${ }^{62}$ Cfr. Hudlet, Karen (Comp.), Compendio de información que presentan la Coalición de Organizaciones de la Sociedad Civil al Grupo de Trabajo sobre Empresas y Derechos Humanos de la ONU, Centro de Información sobre Empresas y Derechos Humanos, México, 2016, pp. 8 y 9.

${ }^{63}$ Ochoa Reza, Enrique, La reforma al sector eléctrico, Nostra ediciones, México, 2015, p. 109.

${ }^{64}$ Cfr. Art. 117 LIE y Art. 118 de la LH.
} 
Tanto en la industria eléctrica como en el sector de hidrocarburos se establece la obligación de contar con evaluaciones de impacto social, que deberán contener la identificación, caracterización, predicción y valoración de los impactos sociales, así como de las medidas de mitigación correspondientes y al respecto, la autoridad administrativa correspondiente deberá pronunciarse ${ }^{65}$.

En complemento de lo anterior, la LIE señala que con el objeto de salvaguardar los intereses y derechos de los pueblos indígenas se deberán llevar a cabo las consultas y cualquier otra medida necesaria para su salvaguarda ${ }^{66}$; por su parte, la LH establece que cuando se pretendan desarrollar trabajos de infraestructura en materia de hidrocarburos, la Secretaría de Energía será la responsable de realizar la consulta previa, libre e informada, así como cualquier otra actividad necesaria para salvaguardar los derechos e intereses de los pueblos indígenas ${ }^{67}$.

Los procedimientos de consulta, en los cuales podrán intervenir tanto autoridades federales, las EPEs, los Órganos Reguladores Coordinados en Materia Energética (ORCME) ${ }^{68}$ y deberán realizarse libres de coacción, proporcionando información vasta, veraz y culturalmente pertinente a los pueblos y comunidades indígenas asociados al proyecto, con el fin de alcanzar un acuerdo u obtener el consentimiento libre e informado ${ }^{69}$, debiéndose observar los principios rectores de buena fe, libertad, información, pertinencia cultural, transparencia, acomodo y razonabilidad, cumpliendo además con los estándares nacionales e internacionales de la materia ${ }^{70}$.

Las fases mínimas que deberán integrar a los procedimientos de consulta son:

a) Plan de consulta,

b) Acuerdos previos,

c) Informativa,

d) Consultiva,

e) Deliberativa y,

f) Seguimiento de acuerdos ${ }^{71}$.

\footnotetext{
${ }^{65}$ Cfr. Art. 120 LIE y Art. 121 LH.

${ }^{66}$ Cfr. Art. 118 LIE

${ }^{67}$ Cfr. Art. 120 LH.

${ }^{68}$ Bajo esta denominación se refiere conjuntamente a la Comisión Reguladora de Energía y a la Comisión Nacional de Hidrocarburos, que con la Agencia Nacional de Seguridad Industrial y de Protección al Medio Ambiente del Sector Hidrocarburos representan a los reguladores sectoriales especializados. Los ORCME aunque dotados de personalidad y de autonomía técnica y de gestión, pertenecen a la Administración Pública Centralizada Federal, en términos de lo dispuesto por el Art. 2, fracción III de la Ley Orgánica de la Administración Pública Federal, publicada en el DOF el día 29 de diciembre de 1976, consultable en http://www.diputados.gob.mx/LeyesBiblio/pdf/153_190517.pdf

${ }^{69}$ Cfr. Art. 91 del Reglamento de la LIE (RLIE), publicado en el DOF el día 31 de octubre de 2014, consultable en http://www.diputados.gob.mx/LeyesBiblio/regley/Reg_LIE.pdf; así como el Art. 86 del Reglamento a la LH (RLH), publicado en el DOF el 31 de octubre de 2014, consultable en http://www.diputados.gob.mx/LeyesBiblio/regley/Reg_LIE.pdf

${ }^{70}$ Cfr. Art. 86 del RLIE y Art. 91 del RLH.

${ }^{71}$ Cfr. Art. 92 del RLIE y Art. 87 del RLH.
} 
Tratándose de la propiedad ejidal, los mecanismos de consulta operarán acorde a lo regulado por la LA y la forma en que su órgano de gobierno toma las decisiones ante la Asamblea Ejidal, con la asesoría de la Procuraduría Agraria.

Para el caso de la industria minera, tal y como se señaló en líneas precedentes, siempre tendrán derecho de preferencia las comunidades indígenas para la obtención de la concesión, e incluso el derecho de igualar la mejor oferta en el concurso respectivo.

En todo caso, insistimos el objetivo de la consulta previa es llegar a acuerdos y obtener el consentimiento de los afectados para el desarrollo de la infraestructura.

\section{3. ¿Qué sigue de la consulta previa?}

La industria energética y minera se consideran causas de utilidad pública, por lo que siempre está dentro de las posibilidades de la Administración Pública emitir una declaratoria de utilidad pública y con ello, proceder a la expropiación ${ }^{72}$.

Incluso, a la luz del marco jurídico configurado a partir de la reforma energética, las actividades de exploración y extracción del petróleo y demás hidrocarburos, así como el servicio público de trasmisión y distribución de energía eléctrica, se consideran estratégicas para el Estado mexicano, además de tasarse como de interés social y orden público ${ }^{73}$, por lo que de acuerdo al propio texto constitucional tendrán preferencia sobre cualquier otra actividad que implique el aprovechamiento de la superficie y del subsuelo de los terrenos afectados a aquéllas ${ }^{74}$, previéndose en la legislación secundaria los términos y las condiciones generales de la contraprestación que se deberá cubrir por la ocpuación y afectación superficial o, en su caso, la indemnización respectiva ${ }^{75}$.

Sin embargo, la legislación en materia energética establece además que tratándose de la afectación superficial o constitución de servidumbres legales para el desarrollo de la industria se podrá negociar con los propietarios o titulares de los terrenos, bienes o derechos reales, ejidales o comunales, la contraprestación y los términos y condiciones por el uso, goce o afectación de los mismos ${ }^{76}$, pero precisamente por esta misma razón de utilidad pública, no se deja al simple acuerdo de voluntades y deberán ser notificados a las autoridades correspondientes para su vigilancia y aprobación (de hecho, previo al inicio de negociaciones, deberán notificar a la Secretaría del Desarrollo Agrario, Territorial y Urbano (SEDATU) de tal situación) ${ }^{77}$. Adicionalmente, los acuerdos entre las partes, deberán ser

\footnotetext{
${ }^{72}$ Cfr. Art. 1 de la Ley de Expropiación, así como otras disposiciones específicas contenidas en la LIE, la LH y la LM.

${ }^{73}$ Los conceptos indeterminados como orden público e interés social son recurso frecuente en el derecho mexicano. A mayor abundamiento, Cfr. Béjar Rivera, Luis José, El acto administrativo y su finalidad, Reimp., Porrúa, México, 2016, pp. 39-52.

${ }^{74}$ Un ejemplo de ello, lo encontramos en el Art. 6, párrafo segundo de la LM.

${ }^{75}$ Cfr. Art. Octavo Transitorio de la Reforma Energética.

${ }^{76}$ Cfr. Art. 73 LIE y Art. 100 LH

${ }^{77}$ Cfr. Art. 78 LIE y Art. 101 LH.
}

Anи Fac Der UDC, 2018, 22:1-20 
presentados ante Juez de Distrito en Materia Civil o Tribunal Unitario Agrario para su validación y elevación a cosa juzgada ${ }^{78}$.

En caso de falta de acuerdos, la autoridad podrá imponer la constitución de una servidumbre legal ${ }^{79}$.

\section{CONSIDERACIONES FINALES}

Es una realidad la necesidad imperante en México de un mejor aprovechamiento de los energéticos, así como de la industria minera, sin embargo, este aprovechamiento se debe realizar con absoluto apego a la legalidad en un sentido amplio, es decir, no solo al sistema de derecho interno, sino a toda la normatividad internacional a la que se ha obligado nuestro país, toda vez que al menos los tratados internacionales de los que México sea parte, forman parte del bloque constitucional de Derechos Humanos ${ }^{80}$, y están por tanto sujetos a control de convencionalidad.

En este sentido, la consulta previa a los pueblos indígenas, regulada ya por el Convenio 169 de la OIT era uno de los reclamos pendientes en nuestro país. Con la reforma energética se incorporan a nuestro sistema jurídico los procedimientos de consulta previa para el uso de terrenos pertenecientes a los pueblos y comunidades indígenas, ya que su contenido parece encontrarse alineado con lo que mandata el Convenio de referencia, aunque desafortunadamente la normatividad a nuestro juicio al día de hoy resulta insuficiente, toda vez que lo establecido para el sector energético no encuentra reflejo en otros ámbitos normativos, como es el caso de la minería.

La Recomendación 27/2016 de la CNDH nos parece que es un instrumento que resulta muy útil de cara a legislar de forma expresa sobre esta figura, aunque es necesario apuntar que eleva, tal vez demasiado e innecesariamente, el estándar de cumplimiento al exceder de la mera consulta previa, para requerir ahora el consentimiento libre e informado.

Tampoco debemos perder de vista que la afectación no solo se da sobre las comunidades indígenas, sino que también puede afectar a las comunidades ejidales, cuyas reglas de autodeterminación y regulación provienen ya de una normatividad que requiere muchos ajustes de cara a las necesidades actuales.

Por otra parte, en materia minera no está regulada la consulta previa, aunque si se establece el derecho de preferencia que los pueblos indígenas tienen sobre las concesiones mineras frente a otros posibles concesionarios, excepto en materia energética, en cuyo caso, a pesar de la vocación minera de los terrenos se dará preferencia al sector energético, salvo que pudieran coexistir; pero en estos casos, el derecho de preferencia no prevalece y se tienen que ceñir a las reglas de consulta previa contenidas en la legislación energética.

\footnotetext{
${ }^{78}$ Cfr. Art. 78 LIE y Art. 105 LH.

${ }^{79}$ Cfr. Art. 82 LIE y Art. 106 LH.

${ }^{80}$ Cfr. Art. $1^{\circ}$ CPEUM.
} 
En pocas palabras, aunque consideramos que resulta un gran avance el incorporar a la legislación energética la consulta previa, nos parece que todavía estamos cortos en cuanto a la forma en que debe ser regulada y establecer las reglas del juego, estableciendo un sano balance entre las necesidades de interés general y los derechos de los pueblos indígenas y las comunidades ejidales. En este sentido, la Recomendación de la CNDH no parece abonar al logro de este equilibrio.

Consideramos también indispensable que se regule la figura de la consulta previa para la industria minera, pues aunque la legislación sectorial sí establece algunas garantías para los pueblos indígenas; en esta materia México tiene que adecuarse a los estándares internacionales, pues sin duda, las comunidades autóctonas se ven claramente afectadas por esta industria y los mecanismos existentes resultan insuficientes para garantizar el absoluto respeto a sus derechos.

La justicia todavía no ha tenido pronunciamientos obligatorios al respecto, apenas si encontramos un par de tesis aisladas en relación a la consulta previa y podemos apuntar que recientemente nuestra SCJN atrajo un juicio de amparo promovido en el año 2015 por una comunidad zapoteca en Juchitán de Zaragoza, en el Estado de Oaxaca, cuyos integrantes argumentan que su derecho a consulta previa fue violado en virtud del establecimiento de un Parque eólico con 132 torres por la compañía Eólica del Sur, y una serie de ONGs consideran que con el fallo de la SCJN se podrá generar un precedente que sea un parteaguas en la materia ${ }^{81}$. Al día de hoy no está resuelto, por lo que permanece subiudice.

Sin duda, el que ya esté regulada la figura de la consulta previa en nuestra legislación energética es un gran avance, sin embargo, en cuanto a su realidad operativa, todavía tendremos que esperar los pronunciamientos de los tribunales y el comportamiento de las autoridades administrativas.

\section{BIBLIOGRAFÍA}

Béjar Rivera, Luis José, Curso de Derecho Administrativo, Novum, México, 2012. México, 2016.

Gutiérrez Rivas, Rodrigo y Emanuelli, María Silvia (Coordinadores), Protocolo de Actuación para quienes imparten justicia en casos relacionados con Proyectos de Desarrollo e Infraestructura, Suprema Corte de Justicia de la Nación, México 2014. Hudlet, Karen (Comp.), Compendio de información que presentan la Coalición de Organizaciones de la Sociedad Civil al Grupo de Trabajo sobre Empresas y Derechos Humanos de la ONU, Centro de Información sobre Empresas y Derechos Humanos, México, 2016.

\footnotetext{
${ }^{81} \mathrm{Cfr}$. http://www.cemda.org.mx/celebra-sociedad-civil-decision-de-la-suprema-corte-de-atraer-el-casosobre-eolica-del-sur/
} 
Melesio Nolasco, Josefina María de la Soledad, Megaproyectos y derechos humanos de los pueblos indígenas, Comisión Nacional de los Derechos Humanos, México 2014.

Moreno Castillo, Luis Ferney \& Hernández Mendible, Víctor Rafael (Coords.), Derecho de la Energía en América Latina, t. II, Universidad Externado de Colombia, Bogotá, 2017.

Ochoa Reza, Enrique, La reforma al sector eléctrico, Nostra ediciones, México, 2015.

Rodríguez Garavito, César (Ed.), "La consulta previa a pueblos indígenas: los estándares del derecho internacional", en Programa de Justicia Global y Derechos Humanos, Documentos No. 2, 2009, consultable en http://www.libertadciudadana.org/archivos/Biblioteca\%20Virtual/Documentos\%20I nformes\%20Indigenas/Documentos\%20Internacionales/Derecho/Consulta\%20Previ a\%20a\%20Pueblos\%20Indigenas \%20Los \%20Estandares \%20del\%20Derecho\%20I nternacional\%202009.pdf

Ruiz-Massieu, Mario, Derecho Agrario, IIJ-UNAM, México, 1990.

Sieder, Rachel, Pueblos indígenas y derecho(s) en América Latina, consultado en http://www.rachelsieder.com/wp-content/uploads/2014/el-derecho-en-americalatina-rachel_sieder.pdf. 